\title{
Analyzing the Interaction of Andrographolide and Neoandrographolide, Diterpenoid Compounds From Andrographis Paniculata (Burm.F) Nees, to Cyclooxygenase-2 Enzyme by Docking Simulation
}

\author{
Jutti Levita $^{1,2^{*}}$, Enade P. Istyastono ${ }^{2}$, As'ari Nawawi ${ }^{1}$, Abdul Mutholib ${ }^{3}$, Iwan J.P.

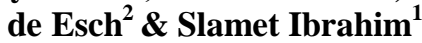 \\ ${ }^{1}$ School of Pharmacy, Institut Teknologi Bandung, \\ Jl. Ganesha 10 Bandung 40116, West Java, Indonesia \\ Email: 1a_via63@yahoo.com; nawawi@fa.itb.ac.id; sibrahim@fa.itb.ac.id \\ ${ }^{2}$ Faculteit der Exacte Wetenschappen, Vrije Universiteit, De Boelelaan 1083, 1081 HV, \\ Amsterdam, The Netherlands, Email: i.de.esch@few.vu.nl; epo200@few.vu.nl \\ ${ }^{3}$ BATAN Serpong, Indonesia, email: mutalib@batan.go.id
}

\begin{abstract}
Cyclooxygenase (COX), an enzyme involved in the conversion of arachidonic acid to prostaglandins, exists in two isoforms, which are COX-1 and COX-2. Despite the similarities of COX-1 and COX-2, the two isoforms show subtle differences in amino acid composition at the active sites. Since COX-1 has isoleucine, a bulkier amino acid at position 523 than COX-2's valine, it allows COX-2 to have a larger space in its active site. Andrographolide reduces COX-2 expression induced by PAF and fMLP in HL60/neutrophils. Neoandrographolide inhibits COX-2 expression at the translational level. The purpose of this study is to examine the binding modes of andrographolide and neoandrographolide against COX-1 and COX-2 in terms of hydrogen bonds and docking energy, to understand their antiinflammatory property. The docking simulation indicates that both andrographolide and neoandrographolide are able to be located in the COX-2's binding pocket but not in the COX-1's. It confirms that COX-1's binding pocket is smaller than COX-2's. Based on this study, both andrographolide and neoandrographolide show selective inhibitory property to COX-2. Their selectivity are due to their specific interaction with Arg 513 in the binding pocket of COX-2, which is also shown by SC-558, a COX-2 selective inhibitor.
\end{abstract}

Keywords: COX; andrographolide; neoandrographolide; docking simulation.

\section{Introduction}

Prostaglandin synthase catalyzes two separate reactions; the first being the cyclooxygenase function, which is the addition of molecular oxygen to arachidonic acid to form the unstable $\mathrm{PGG}_{2}$, and the second the further conversion of $\mathrm{PGG}_{2}$ to the more stable $\mathrm{PGH}_{2}$, which catalyzes the conversion of

Received March $2^{\text {nd }}, 2009$, Revised June $15^{\text {th }}, 2009$, Accepted for publication September $8^{\text {th }}, 2009$. 
hydroperoxide group to hydroxyl group by hydroperoxide function. Hence this COX enzyme performs a critical initial reaction in the arachidonic metabolic cascade leading to the formation of prostaglandins, thromboxan and prostacyclin [1].

Two known isoforms of COX, named COX-1 and COX-2, show distinct expression patterns and distinct biological activities. COX-1 is constitutively expressed protein formed in many different cells and responsible for the production of prostaglandins. COX-2 is rapidly up-regulated at inflammatory sites and appeared responsible for the formation of proinflammatory prostanoids. COX-1, meanwhile, appeared to shoulder the responsibility for the production of physiologically relevant prostanoids such as those in the stomach and platelets [2,3,4]. In inflammatory processes COX-2 is overexpressed [5]. The two isoforms are $63 \%$ identical and $77 \%$ similar at amino acids level. Based on the sequence alignment of human COX-1 and COX-2, the major differences are in the $\mathrm{N}$-terminal and $\mathrm{C}$-terminal regions. Structural differences that are responsible for selectivity are believed to be found in the cyclooxygenase active site. One difference is observed at the cyclooxygenase active site, where the isoleucine at position 523 in COX-1 is a valine in COX-2 [1]. Other researchers reported that despite the similarities of COX-1 and COX2 , the two isoforms show subtle differences in amino acid composition at the active sites, i.e. the bulky isoleucine at position 523, while COX-2 has valine, hence resulting a larger space in the active site of COX-2. This difference is believed to induce the selectivity of some inhibitors [2].

The leaves of Andrographis paniculata (Burm.F) Nees had been empirically used to treat inflammations in Indonesia, especially by the Javanese. Andrographolide and neoandrographolide are diterpenoid compounds isolated from Andrographis paniculata (Burm.F) Nees [6]. The two compounds are classified as lactone bicyclic diterpenoids, which have been reported to have antiinflammatory activity. Andrographolide reduces COX-2 expression induced by PAF and fMLP in HL60/neutrophils, as reported by Maria A. Hidalgo et al [7]. Neoandrographolide inhibits COX-2 expression at the translational level, as reported by J. Liu and colleagues from Shanghai University [8]. In this paper the binding modes of andrographolide and neoandrographolide against COX enzymes in terms of hydrogen bonds and docking energy were studied to understand their antiinflammatory activity.

\section{Results}

Stochastic conformational search using molecular mechanics method of MOE2007.09.02 software was performed to find the individual energy minimum state. The molecular mechanics considers the atomic composition of a molecule 
to be a collection of masses interacting with each other via harmonic forces. As a result of this simplification, molecular mechanics is a relatively fast computational method practicable for small molecules as well as for larger molecules and even oligomolecular systems. This search resulted eleven conformers for andrographolide and seven conformers for neoandrographolide. Both molecules are flexible due to their many single bonds. The flexibility of andrographolide and neoandrographolide leads to their tendency to interact with their targets more easily.

The molecular surfaces of andrographolide and neoandrographolide showed that both molecules are likely to form hydrogen bonds considering their carbonyl, lactone and hydroxyl (Figure 1).

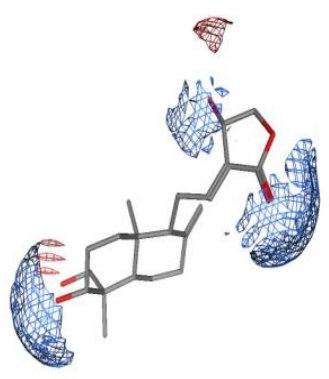

(a)

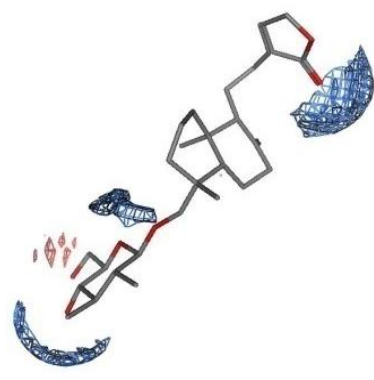

(b)

Figure 1 Electrostatic map of (a) andrographolide (b) neoandrographolide. The blue color indicated electron-donating area.

Table 1 Similarity study.

\begin{tabular}{lcccccc}
\hline \multicolumn{1}{c}{$\begin{array}{c}\text { Aligned } \\
\text { compounds }\end{array}$} & $\begin{array}{c}\text { Similarity terms } \\
\text { (Default setting) }\end{array}$ & \multicolumn{3}{c}{$\begin{array}{c}\text { Similarity terms } \\
\text { (aromaticity, acid/base, } \\
\text { hydrophobe area, polar hydrogens, } \\
\text { log P, molar refractivity, partial } \\
\text { charge, volume) }\end{array}$} \\
\cline { 2 - 7 } & $\mathrm{F}$ & $\mathrm{S}$ & $\mathrm{dU}$ & $\mathrm{F}$ & $\mathrm{S}$ & $\mathrm{dU}$ \\
\hline $\begin{array}{l}\text { Andographolide } \\
\text { and flurbiprofen }\end{array}$ & 221.1373 & 286.4565 & 0.0000 & 126.2464 & 194.7328 & 2.7613 \\
$\begin{array}{l}\text { Andrographolide } \\
\text { and SC-558 }\end{array}$ & 157.5603 & 214.5916 & 0.0000 & 110.4931 & 166.8766 & 0.0000 \\
\hline
\end{tabular}

$\mathrm{F} \quad=$ similarity

$\mathrm{S} \quad=$ objective function

$\mathrm{dU}=$ the difference between lowest potential energy and the global minimum 
Similarity study was performed by using flexible alignment tool which aligned two or more molecules with several similarity terms and weighing parameters. We have chosen aromaticity, acid/base, hydrophobe area, polar hydrogens, lipophilicity (log P value), molar refractivity, partial charge, and volume as the similarity parameters, and all parameters were weighed 1 (Table 1 and Figure 2).

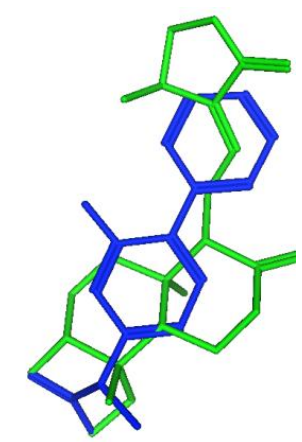

(a)

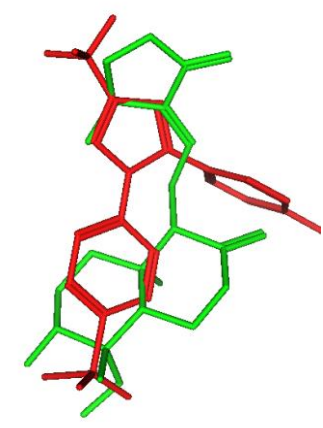

(b)

Figure 2 Flexible alignment of andrographolide (green) and flurbiprofen (blue) andrographolide (green) and SC-558 (red).

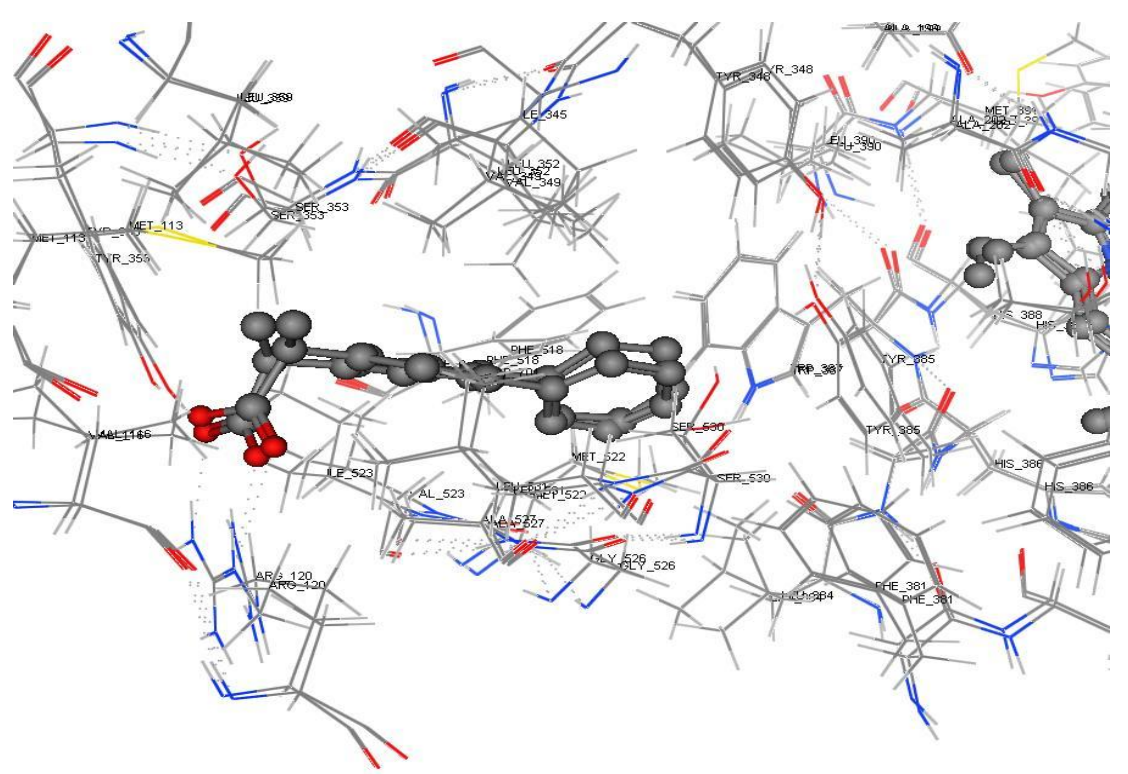

Figure 3 Alignment of COX-2 (code 3PGH) and COX-1 (code 1EQH) crystal structures represent the same site in which their non-selective inhibitor, flurbiprofen was co-crystallized. 
Andrographolide shows similarity to SC-558 than to flurbiprofen (dU value of andrographolide and SC-558 at different settings is 0.0000). The smaller the value of $\mathrm{F}$ and $\mathrm{S}$ indicates similarity of the compounds aligned.

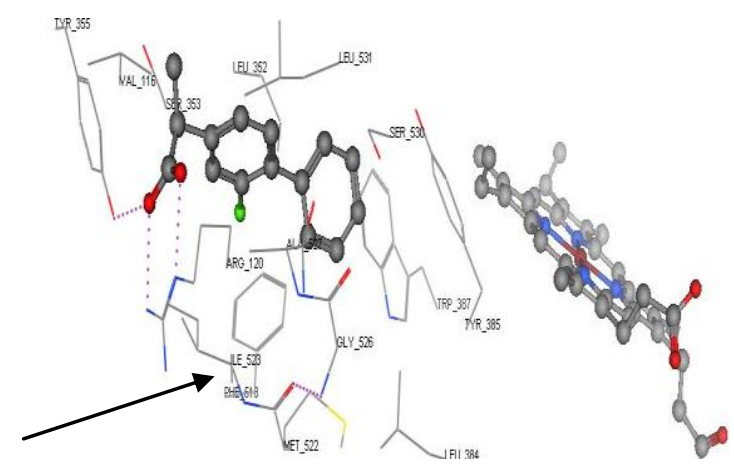

(a)

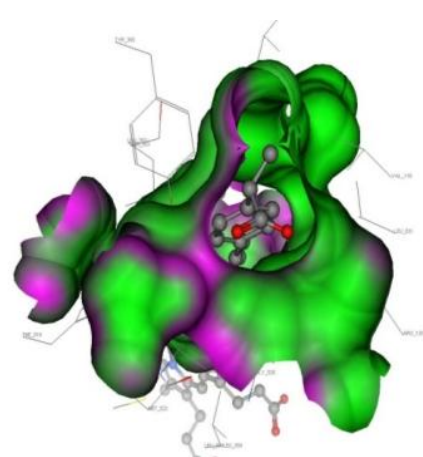

(b)

Figure 4 (a). Flurbiprofen's interaction in the binding site of COX1. The arrow showed Ile 523, specific amino acid in COX1 binding site. (b). Flurbiprofen in the binding site of COX1.

Analysis of binding sites in COX-1 and COX-2 enzymes were performed by aligning crystal structures of the two enzymes (code 1EQH and 3PGH), in which flurbiprofen was co-crystallized, and examining the site where it was bound. Amino acid residues involved in the binding site of COX1 were His 90, Arg 120, Val 349, Leu 352, Ser 353, Tyr 355, Trp 387, Phe 518, Met 522, Ile 523, Gly 526, Ala 527, Ser 530, Leu 534, while in COX2 were His 90, Leu 117, Val 349, Leu 352, Ser 353, Tyr 355, Trp 387, Ala 516, Phe 518, Met 522, Val 523, Gly 526, Ala 527, Ser 530, Leu 534. In both enzymes, flurbiprofen lies exactly in the same site (Figure 3). The ligand formed two hydrogen bonds with Arg 120 and Tyr 355 (Figure 4). Further analyze of COX-2 interaction with its selective inhibitor SC-558 (code 1CX2) were performed. SC-558 shows hydrogen bond interactions with Arg 513 and Gln 192 (Figure 5). The position of Arg 513 is at the lower side of the pocket, which means that the pocket of COX-2 is larger in size than COX-1's. The interaction of SC-558 with Arg 513 might be important because it makes this ligand selective to inhibit COX-2 activity. 


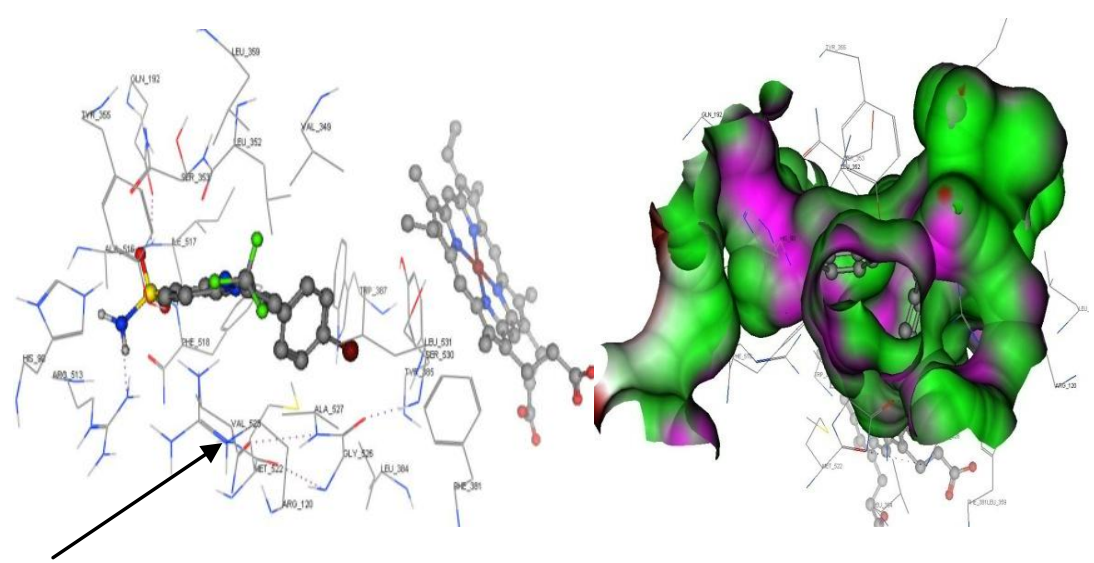

(a)

(b)

Figure 5 (a) SC-558, a selective inhibitor of COX2, interacted with Arg 513 and Gln 192 in the binding site of COX2. The arrow showed Val 523, specific amino acid in COX2 binding site which makes COX2's binding pocket larger in size than COX1's. (b) SC-558 in the binding site of COX2.

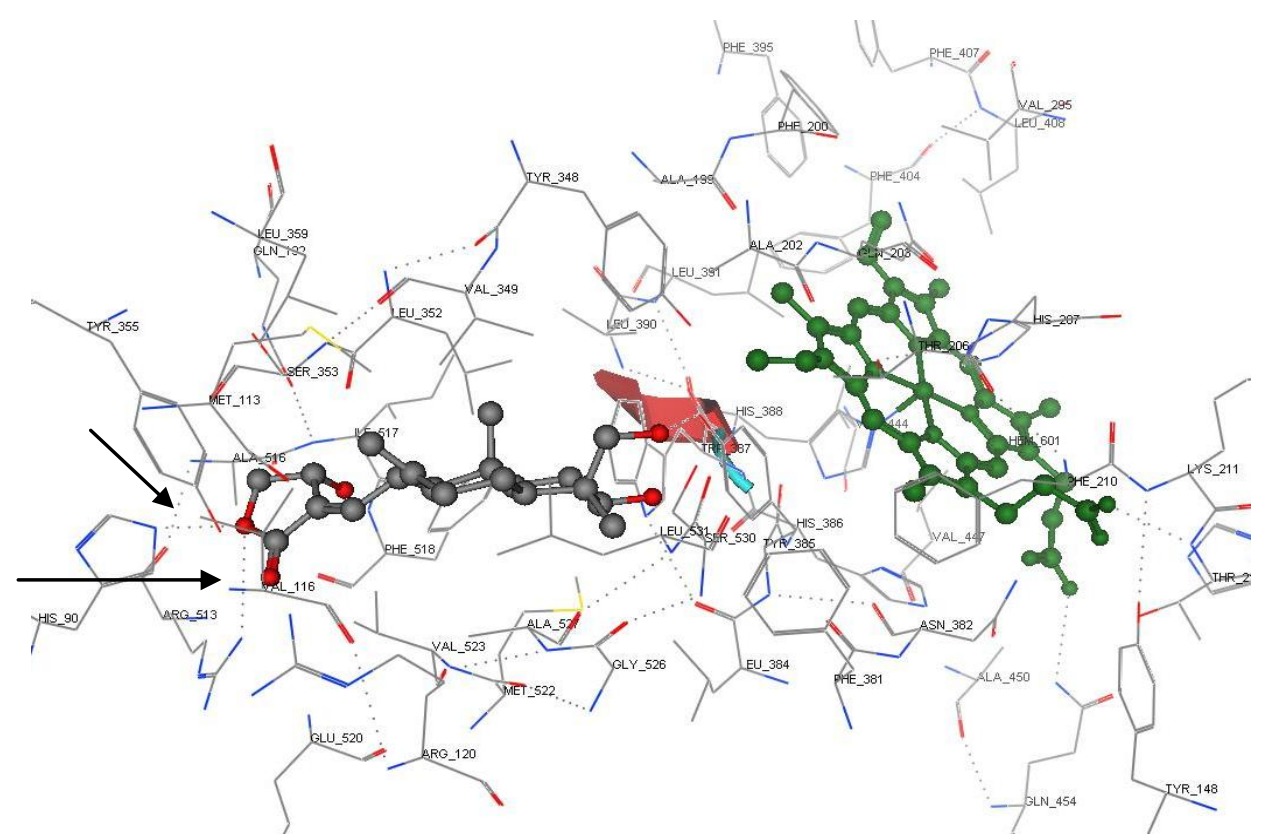

Figure 6 Docking simulation of andrographolide into the binding site of COX2. Hydrogen bonds are showed by black arrows. 


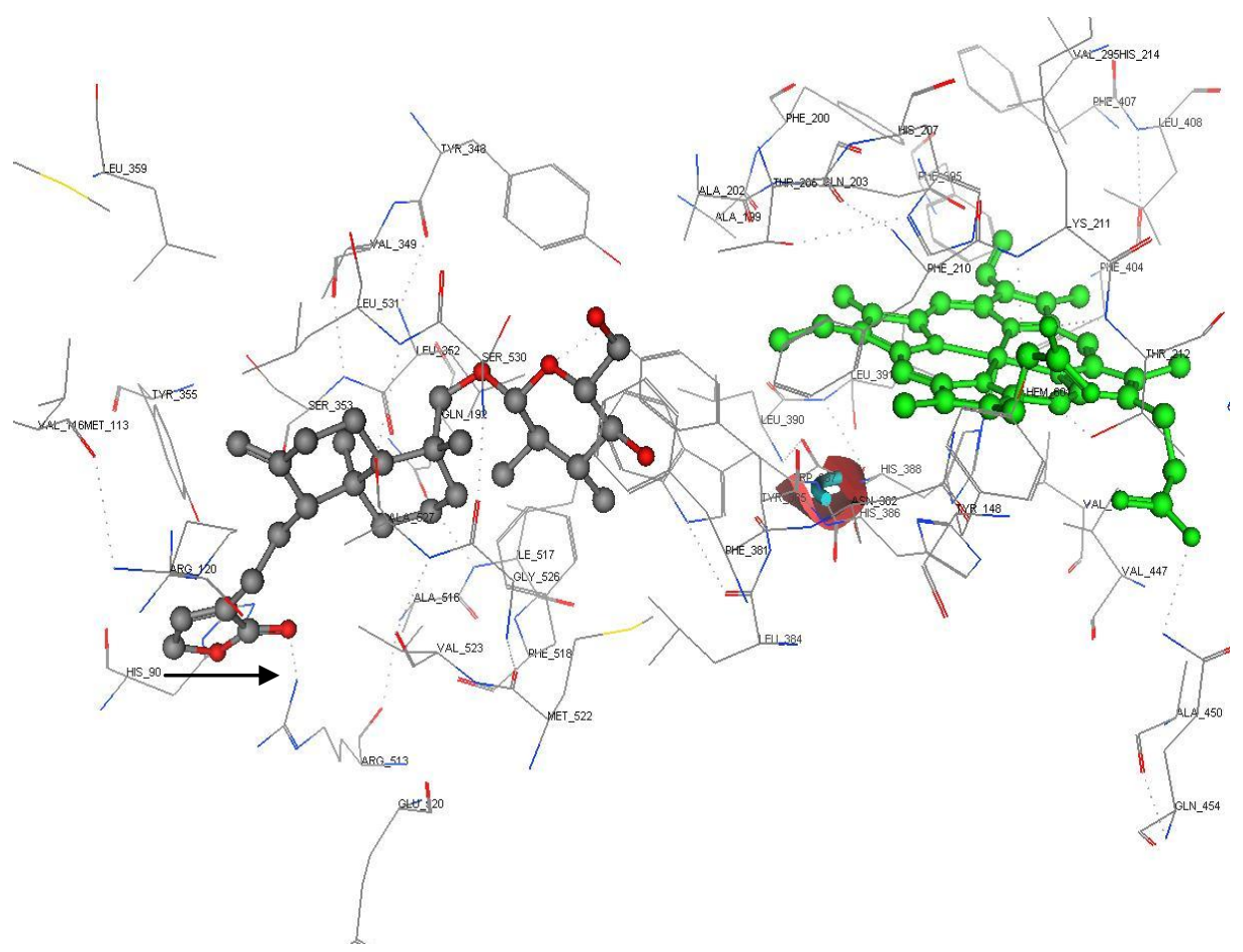

Figure 7 Docking simulation of neoandrographolide into the binding site of COX2. Hydrogen bonds are showed by black arrows.

To identify and visualize the antiinflammatory activity of andrographolide and neoandrographolide, docking calculations were performed using MOE2007.09.02 into the 3D structures of the catalytic site of COX-1 (PDB code: $\mathrm{EQH}$ ) and COX-2 (PDB code: 1CX2) enzymes. Andrographolide and neoandrographolide cannot be docked into the COX-1 binding pocket, probably due to the size of the pocket. This indicates that COX-1 binding pocket is smaller than COX-2. We have tried to force the compounds to be docked into the COX-1 binding pocket (by using AutoDock v.3.0.5 software), but it resulted in large value of inhibition constant, $\mathrm{Ki}$, and positive value in docking energy, which meant that the interactions were not favourable (data was not showed). However, andrographolide shows interaction with the COX-2 binding pocket (Figure 6) through forming hydrogen bonds between oxygen atom in andrographolide's lactone ring (HB acceptor) and hydrogen atom in the Arg 513's amine and His 90's pyridine (HB donors), while neoandrographolide forms hydrogen bonds with Arg 513 (Figure 7). 


\section{Discussion}

Andrographolide and neoandrographolide, diterpenoid compounds from Andrographis paniculata (Burm.F) Nees have been reported to have antiinflammatory activity in vitro. This study has confirmed that this activity might be caused by their interactions with the binding pocket of COX-2 enzymes. Both molecules, which has potential moieties to form hydrogen bonds, e.g. oxygen and hydrogen atoms in the hydroxyl groups and oxygen atom in the lactone ring and carbonyl groups, are shown to form hydrogen bonds with Arg 513, His 90 and other amino acids in the pocket.

We have analyzed the interaction of COX-2 selective inhibitor, SC-558, in the binding pocket of COX-2. The compound, which has smaller size compared to andrographolide and neoandrographolide, shows only one specific interaction in the pocket by forming hydrogen bond with Arg 513. We hypothesized that if any compounds made the same interactions, by means : building hydrogen bonds with Arg 513 (which is located at the lower site of the pocket), then the compounds would be potential as selective inhibitors of COX2. In this study, it was confirmed by employing molecular modeling approach using docking simulation of the two compounds into both COX-1 and COX-2 binding pockets. Andrographolide and neoandrographolide are more favourable to interact with COX-2's binding pocket than SC-558 (Figure 6 and 7), which is proven by the formation of hydrogen bonds and the value of docking energy. Hydrogen bonds are formed between oxygen atom in andrographolide's lactone ring (HB acceptor) and hydrogen atom in the Arg 513's amine and His 90's pyridine (HB donors), while the value of docking energy is $-10.64 \mathrm{kcal} / \mathrm{mol}$. Neoandrographolide's hydroxyl which is attached at its lactone ring forms hydrogen bonds with Arg 513's amine, while its docking energy value is -7.77 $\mathrm{kcal} / \mathrm{mol}$. It should be noted that SC-558 only forms one hydrogen bond with Arg 513.

This study might be useful to visualize and understand the interactions of andrographolide and neoandrographolide to cyclooxygenase enzymes at molecular level.

\section{Conclusions}

The docking simulation indicates that both andrographolide and neoandrographolide can not interact with COX-1's binding pocket, probably due to the size of the pocket. Andrographolide and neoandrographolide show interactions with COX-2's binding pocket by forming hydrogen bonds to Arg 513 , His 90 and other amino acids in the pocket. Their selectivity is due to their 
specific interactions with Arg 513 in the binding pocket of COX-2, which is also shown by SC-558, a COX-2 selective inhibitor.

\section{$5 \quad$ Experimental}

\subsection{Data Preparation}

The x-ray crystallographic 3D structures of COX-1 (PDB code: 1EQH ) and COX-2 (PDB codes: 1CX2 and 3PGH) were downloaded from online Protein Data Bank (http://www.rcsb.org/pdb/). 2D and 3D structures of andrographolide and neoandrographolide were built using Molecular Operating Environment (MOE-2007.09.02).

Docking andrographolide and neoandrographolide to COX-1 and COX-2 enzymes were carried out using MOE-2007.09. 02.

\subsection{Molecular Modeling}

MOE-2007.09.02 was applied to build the ligands. Energy minimization to each molecule was carried out by using molecular mechanics forcefield MMFF94x. Stochastic conformational search with molecular mechanics method was performed to find the lowest energy conformers. Molecular surfaces of andrographolide and neoandrographolide, by means their electrostatic maps, were also computed. Hydrogens were added to all COX enzymes PDB crystal structures followed by calculating the partial charges by using Amber 99. Site finder tool was performed to each crystal structure molecule to predict the most likely sites for the ligand-enzyme interactions. MOE sequence editor was used to analyze the amino acid sequences in the enzyme. Flexible alignment was applied to analyze the similarity of andrographolide and flurbiprofen and SC558.

\subsection{Analysis of Binding Site Residues}

$\mathrm{X}$-ray crystallographic 3D structures of COX-1 (PDB code: 1EQH) and COX-2 (PDB code : 3PGH), both complexed with the non-selective inhibitor flurbiprofen, were studied to obtain information about their differences, which in this case refered to the amino acid residues in the binding sites. A comparative study using 1CX2, an x-ray crystallographic structure of $\mathrm{COX} 2$ complexed with a selective inhibitor SC-558, was performed to understand the ligand's selective inhibition property to COX2. 


\subsection{Ligand-Protein Docking}

The prediction of interaction of andrographolide and neoandrographolide to COX-2 is the main challenge to understand their antiinflammatory property. The goal is to take the 3D coordinates of the enzyme and its complexed ligand (SC-558) which has been known as a selective inhibitor to COX-2, and to simulate the docking of our compounds into the site in which SC-558 was cocrystallized. The interactions between SC-558 and COX-2 binding site was analyzed and compared with andrographolide and neoandrographolide's.

\section{Acknowledgements}

This work and visit to Vrije Universiteit Amsterdam The Netherlands were financially supported by the goverment of The Republic of Indonesia.

The authors have declared no conflict of interest.

\section{References}

[1] J.K., Gierse, J.J., McDonald, S.D., Hauser, S.H., Rangwala, C.M., Coboldt, and K., Seibert, The Journal of Biological Chemistry, 1996, 271 (26): 15810-15814.

[2] G.F., Fabiola, L., Damodharan, V., Pattabhi, and K., Nagarajan, Current Science, 2001, 80: 26-34.

[3] J.R., Vane, J. Physiol. Pharmacol. 2000, 51 : 573-586.

[4] J.A., Mitchell., T.D., Warner, Br. J. Pharmacol., 1999, 128 : 1121-1132

[5] S.P., Dilber, S.Lj., Dobric, Z.D., Juranic, B.D., Markovic, S.M., Vladimirov, and I.O., Juranic, Molecules, 2008, 13 : 603-615.

[6] T., Fujita, R., Fujitani, Y., Takeda, Y., Takaishi, T., Yamada, M., Kido, and I., Miura, Chem. Pham. Bull., 1984, 32 (6) : 2117-2125.

[7] M.A., Hidalgo, et al, British Journal of Pharmacology, 2005, 144: 680685.

[8] J., Liu, Z.T., Wang, L.L., Ji, B.X., Ge, Molecular and Cellular Biochemistry, 2007, 298(1-2): 49-57. 